\title{
Synthesis of 4-Imidazolidinones from Diamides and Ethynyl Benzio- doxolones vis Double Michael Addition: Ethynyl Benziodoxolones as Electrophilic Ynol Synthons
}

\author{
Ayaka Shimizu ${ }^{\mathrm{a}}$, Atsushi Shibata ${ }^{\mathrm{a}}$, Hiroyoshi Esaki ${ }^{\mathrm{b}}$, Kazuaki Fukushima ${ }^{\mathrm{b}}$, Norihiro Tada ${ }^{\mathrm{a}}$, and \\ Akichika Itoh ${ }^{\mathrm{a}^{*}}$ \\ ${ }^{a}$ Gifu Pharmaceutical University, 1-25-4 Daigaku-nishi, Gifu 501-1196, Japan \\ ${ }^{b}$ Hyogo College of Medicine, 1-1 Mukogawa-cho, Nishinomiya, Hyogo 663-8501, Japan \\ *Email: esaki@hyo-med.ac.jp; ntada@gifu-pu.ac.jp; itoha@gifu-pu.ac.jp
}

Supporting Information Placeholder

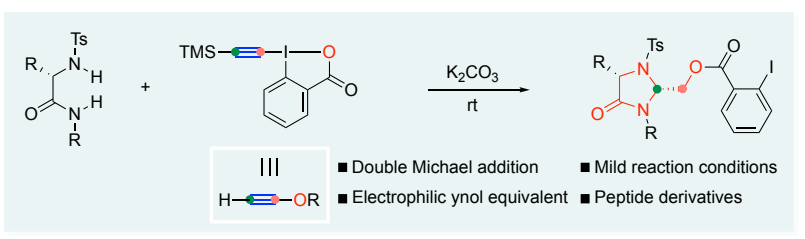

\begin{abstract}
The moiety of 4-imidazolidinone is an important structural motif in organic synthesis and medicinal chemistry. We present the synthesis of 4-imidazolidinones from various diamides with ethynyl benziodoxolones through double Michael addition, which is an unprecedented reaction mode for hypervalent alkynyl iodine compounds. cis-2,5-disubstituted 4-imidazolidinones were diastereoselectively synthesized from amino-acid-derived diamides. Having derivatized the 4-imidazolidinones, several control experiments and density functional theory calculations were conducted to realize mechanistic insight.
\end{abstract}

The 4-imidazolidinone structure is an important motif that exists in many bioactive natural products, such as asperlicin and scedapin $C^{1}$ (Figure 1), particularly where such compounds bear an alcohol moiety at the 2-position of the 4-imidazolidinone. Medicinal and biologically active compounds, such as spiperone and hetacillin, also have the 4-imidazolidinone structure ${ }^{2}$ (Figure 1). Furthermore, the introduction of 4-imidazolidinone structures changes the activity and stability of peptides, such as Leu-enkephalin (Figure 1), vancomycin, and cyclic peptides. ${ }^{3}$ Additionally, 4-imidazolidinones have been used as chiral organocatalysts, ${ }^{4}$ ligands, ${ }^{5}$ and synthetic intermediates. ${ }^{6}$ Accordingly, there are numerous synthetic methods available to access 4-imidazolidinones. ${ }^{7-8}$ The most general and straightforward method is via the condensation of a-amino amides with aldehydes and ketones. ${ }^{9}$ However, this reaction generally requires harsh reaction conditions, such as strong acids, high temperature, and/or excess amounts of aldehyde and ketone plus the removal of water and multiple steps. In 2016, Bode et al. ${ }^{9 a}$ reported the construction of 4-imidazolidinone as a mixture of diastereomers under mild conditions via an aldehyde conjugation reaction using amino hydrazide and amino hydroxamic acid. Furthermore, Raj et al. ${ }^{3 a}$ reported the "CyClick" strategy, which involves the intramolecular construction of 4-imidazolidinones in a cyclic peptide, which enables their construction under mild conditions. Thus, the synthesis of 4-imidazolidinones under mild reaction conditions has potential for use as a chemical biology tool and in terms of its intrinsic synthetic value.

Hypervalent alkynyl iodine compounds have been used for the synthesis of various heterocycles by Michael addition and cyclization via alkylidene carbene or alkyne under mild reaction conditions due to the high electron-withdrawing nature and hyper-leaving-group character of the $\lambda^{3}$-iodanyl group. ${ }^{10-13}$ Furthermore, the chemistry of benziodoxol(on)e reagents has recently attracted considerable interest because of their higher stability compared with their acyclic analogs. ${ }^{14}$ Alkynyl benziodoxolones have also been used for the synthesis of heterocycles (with and without metal catalysts). ${ }^{15-16}$ In 2013, Cossy et al. ${ }^{16 a}$ reported the synthesis of tetrahydropyrazines from diamides using trimethylsilyl ethynyl benziodoxolone (TMS-EBX) in the presence of a strong base through 6-endo-dig cyclization of the ynamide intermediate $^{16 a}$ (Scheme 1A). Furthermore, during the course of our investigations, Miyake et al. ${ }^{16 \mathrm{~b}}$ reported the synthesis of 1,2-dithio-1-alkenes, including benzene-1,2-dithiol as a substrate to benzo-1,4-dithiines, using EBX reagents by intramolecular thiyl radical addition to thiol-substituted vinyl benziodoxolones $^{17-18}$ (Scheme 1B). Recently, due to our interest in the development of the reaction with hypervalent iodine compounds, ${ }^{19}$ we reported the synthesis of cis- $\beta$-amide-vinylbenziodoxolones from sulfonamide, including amino-acid derivatives, with an ethynyl benziodoxolone-chloroform complex. ${ }^{19 a}$ Based on the results of the aforementioned studies, we hypothesized that the reaction of an amino-acid-derived diamide with ethynyl benziodoxolone would afford 4-imidazolidinone by sequential intermolecular and intramolecular double Michael addition, which is, to the best of our knowledge, an unprecedented reaction mode of hypervalent alkynyl iodine compounds (Scheme 1C). After the cyclization, the benziodoxolone may convert to 2-iodobenzoate, which is an atom-economical reaction because 
2-iodobenzoate moiety serves as a versatile handle for further transformations ${ }^{20}$ (Scheme 1C). Double Michael additions have been used for the construction of various cyclic compounds ${ }^{21}$; however, to our knowledge, the synthesis of 4-imidazolidinone by such means has not been reported. Herein, we report straightforward access to 4-imidazolidinones from diamides using TMS-EBX or EBX-MeCN through a double Michael addition reaction and a formal reductive elimination sequence (Scheme 1C). Interestingly, the EBX reagents worked as an electrophilic ynol surrogate, which had umpolung reactivity to a normal nucleophilic ynol ${ }^{22}$ (Scheme 1C).

Figure 1. 4-Imidazolidinone in bioactive natural products, medicine, and a prodrug.

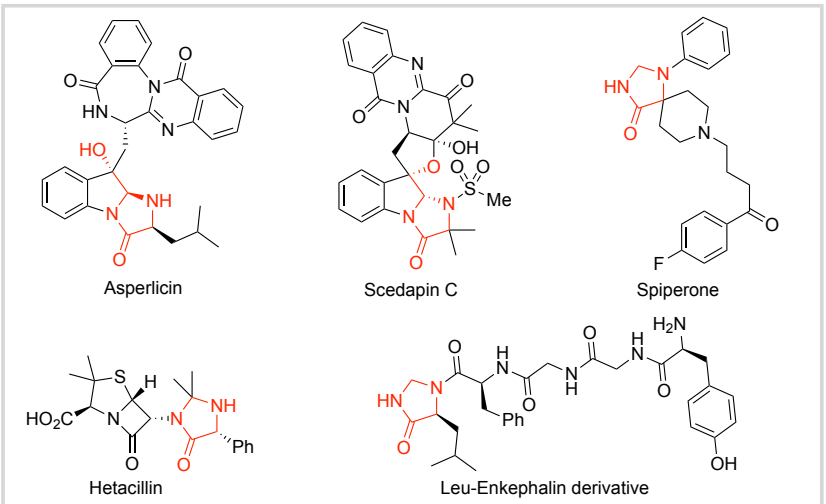

Scheme 1. Previous and this work

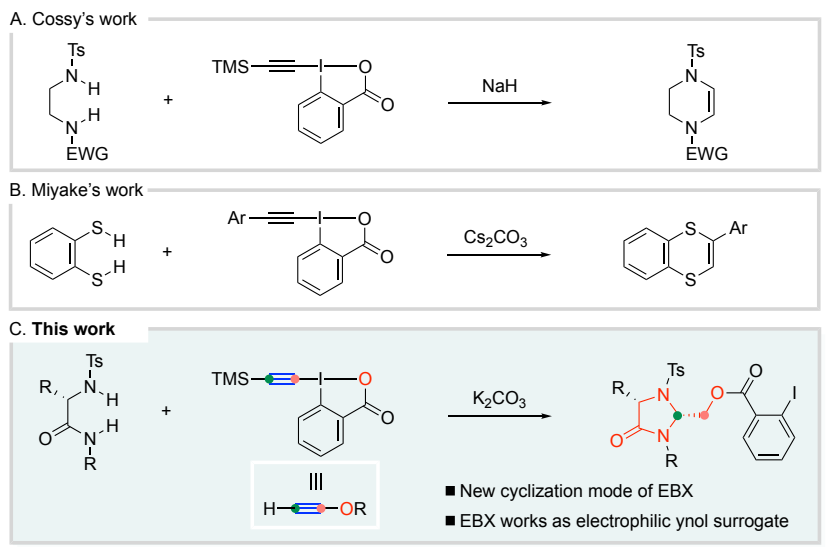

To explore our hypothesis, we initially attempted the reaction with glycine-derived diamide $\mathbf{1 a}$ as the substrate (Table 1). When EBX-MeCN complex (2a), prepared from TMS-EBX (2b) ${ }^{19 \mathrm{e}}$ was used, all solvents gave 4 -imidazolidinone (3a) in moderate to good yields (entries $1-5$ ). However, $2 \mathbf{b}$ gave more solvent-dependent results (entries 6-10). Among the solvents we used, mixed solvent (IPA/ $\mathrm{MeCN}=1: 1$ ) gave the best isolated yield ( $80 \%$ ) with $\mathbf{2 b}$ (entry 7 ). A screening of bases with 2b revealed that sodium carbonate and cesium carbonate also gave the desired product in good yields (entries 11 and 12). However, organic bases gave lower product yields (entries 13 and 14), and in the absence of a base, no reaction occurred (entry 15). Additionally, an increase in the amount of base did not improve the yield for the synthesis of 3a (entries 16 and 17). Consequently, the optimal reaction conditions were identified as follows: treatment of $\mathbf{1 a}$ with $\mathbf{2} \mathbf{b}$ (1.3 equiv.) in the presence of $\mathrm{K}_{2} \mathrm{CO}_{3}$ (0.1 equiv.) in IPA/MeCN $(1: 1 v / v)$ at room temperature for $30 \mathrm{~min}$ (entry 7). Note that 3a was also synthesized at the $1 \mathrm{mmol}$ scale with $84 \%$ yield, indicating the scalability of the reaction (entry 7 ).

With the optimized reaction conditions in hand, we examined the scope and limitations of the reaction system (Scheme 2). Under the optimized conditions, 2-nitrobenzenesulfonamide was transformed into the corresponding product $\mathbf{3 b}$ in low yield (24\%); however, extending the reaction time to $24 \mathrm{~h}$ gave an improved yield (59\%). Conversely, carbamate 1c was recovered unchanged probably because of its low acidity. ${ }^{16 a, 19 a}$ Furthermore, the reactions of bromo-, iodo-, and methyl-substituted aniline derivatives afforded the corresponding products in good yields regardless of the position of the substituent (3d-3h).

Table 1. Study of reaction conditions ${ }^{a}$

\begin{tabular}{|c|c|c|c|c|}
\hline 1a $(0.05 \mathrm{mmol})$ & & 2a: $X=H ; 2$ (2): $X=\mathrm{TMS}$ & $\begin{array}{c}\text { base (equiv) } \\
\text { solvent (1 mL) } \\
\mathrm{rt}, 30 \mathrm{~min}, \mathrm{Ar}\end{array}$ & Pa \\
\hline entry & 2 & base (equiv) & solvent & yield (\%) \\
\hline 1 & $2 a$ & $\mathrm{~K}_{2} \mathrm{CO}_{3}(0.1)$ & IPA & 68 \\
\hline 2 & $2 a$ & $\mathrm{~K}_{2} \mathrm{CO}_{3}(0.1)$ & IPA:MeCN=1:1 & (77) \\
\hline 3 & $2 a$ & $\mathrm{~K}_{2} \mathrm{CO}_{3}(0.1)$ & $\mathrm{MeCN}$ & 68 \\
\hline 4 & $2 a$ & $\mathrm{~K}_{2} \mathrm{CO}_{3}(0.1)$ & $\mathrm{AcOEt}$ & 49 \\
\hline 5 & $2 a$ & $\mathrm{~K}_{2} \mathrm{CO}_{3}(0.1)$ & $\mathrm{CH}_{2} \mathrm{Cl}_{2}$ & 46 \\
\hline 6 & $2 b$ & $\mathrm{~K}_{2} \mathrm{CO}_{3}(0.1)$ & IPA & 0 \\
\hline 7 & $2 b$ & $\mathrm{~K}_{2} \mathrm{CO}_{3}(0.1)$ & IPA:MeCN=1:1 & $(80),(84)^{b}$ \\
\hline 8 & $2 b$ & $\mathrm{~K}_{2} \mathrm{CO}_{3}(0.1)$ & $\mathrm{MeCN}$ & 66 \\
\hline 9 & $2 b$ & $\mathrm{~K}_{2} \mathrm{CO}_{3}(0.1)$ & AcOEt & 12 \\
\hline 10 & $2 b$ & $\mathrm{~K}_{2} \mathrm{CO}_{3}(0.1)$ & $\mathrm{CH}_{2} \mathrm{Cl}_{2}$ & 5 \\
\hline 11 & $2 b$ & $\mathrm{Cs}_{2} \mathrm{CO}_{3}(0.1)$ & IPA:MeCN=1:1 & 78 \\
\hline 12 & $2 \mathrm{~b}$ & $\mathrm{Na}_{2} \mathrm{CO}_{3}(0.1)$ & IPA:MeCN=1:1 & 77 \\
\hline 13 & $2 b$ & $\mathrm{Et}_{3} \mathrm{~N}(0.1)$ & IPA:MeCN=1:1 & 44 \\
\hline 14 & $2 b$ & pyridine $(0.1)$ & IPA:MeCN=1:1 & 0 \\
\hline 15 & $2 b$ & - & IPA:MeCN=1:1 & 0 \\
\hline 16 & $2 b$ & $\mathrm{~K}_{2} \mathrm{CO}_{3}(0.2)$ & IPA:MeCN=1:1 & 79 \\
\hline 17 & $2 b$ & $\mathrm{~K}_{2} \mathrm{CO}_{3}(1.0)$ & IPA:MeCN=1:1 & 79 \\
\hline
\end{tabular}

${ }^{a}$ Reaction conditions: $1 \mathrm{a}(0.05 \mathrm{mmol}), 2(0.065 \mathrm{mmol})$, base, solvent $(1 \mathrm{~mL})$, room temperature, $30 \mathrm{~min}$, argon. Yields were determined by ${ }^{1} \mathrm{H}$ NMR using 1,1,2,2-tetrachloroethane as an internal standard. Numbers in parentheses are isolated yields. ${ }^{b} 1 \mathrm{mmol}$ scale.

Moreover, the reaction proceeded with benzylamine derivatives bearing electron-withdrawing and electron-donating groups $(\mathbf{3 i}, \mathbf{3 j})$. While a furan substituent was also well tolerated (3k), a pyridine substituent resulted in a complex mixture but no corresponding final product was obtained (3l). Pleasingly, dipeptide $1 \mathrm{~m}$ gave the corresponding cis-2,5-disubstituted 4imidazolidine $\mathbf{( 3 m})$ diastereoselectively in moderate yield $(46 \%)$ under the optimized conditions. Interestingly, an improved yield of $3 \mathbf{m}(64 \%)$ was obtained using 1 equiv. of $\mathrm{K}_{2} \mathrm{CO}_{3}$. Furthermore, using EBX-MeCN instead of TMS-EBX gave a better yield of $3 \mathrm{~m}$ ( $46 \%$ vs. $73 \%$ and $64 \%$ vs. $89 \%$ ). This result indicated that unprotected $\mathrm{EBX}-\mathrm{MeCN}$ shows better reactivity to some substrates, which allows addition reactions without deprotection of TMS under mild conditions. Note that no trans2,5-disubstituted 4-imidazolidines were detected via crude ${ }^{1} \mathrm{H}$ NMR. Other dipeptides also gave the corresponding products in moderate to good yields with 1 equiv. $\mathrm{K}_{2} \mathrm{CO}_{3} \quad(\mathbf{3 n}-\mathbf{3 r})$. 
Furthermore, other functionalities, such as indole, amide, and ester groups, were well tolerated. 1r gave the many undetermined by-products and a low isolated yield (25\%) of 3r. However, the isolated yield of $\mathbf{3 s}$ was diminished in the separation step with preparative TLC, probably because of the high polarity of 3s. Importantly, 3n was determined to have an ee of $>99 \%$ by HPLC using CHIRALPAK IG. Furthermore, tripeptide $1 \mathrm{~s}$ also gave $3 \mathrm{~s}$ in moderate yield using 1 equiv. of $\mathrm{K}_{2} \mathrm{CO}_{3}$. Interestingly, six- and seven-membered rings were not detected by ${ }^{1} \mathrm{H}$ NMR when asparagine, glutamine, and glutamic acid derivatives were used (3q-3s). Furthermore, bis-tosyl-ethylenediamine gave the corresponding product (3t), indicating that this reaction mode is not limited to the synthesis of 4-imidazolidinones. Conversely, the Ts-Boc-ethylenediamine gave the labile cis- $\beta$-substituted vinylbenziodozolone (3u) in $61 \%$ yield along with an undetermined impurity. This result could indicate that tosylamide initially adds to the $\beta$-position of EBX to produce the vinyl

Scheme 2. Scope and limitations of the synthesis of 4-imidazolidinone $^{a}$

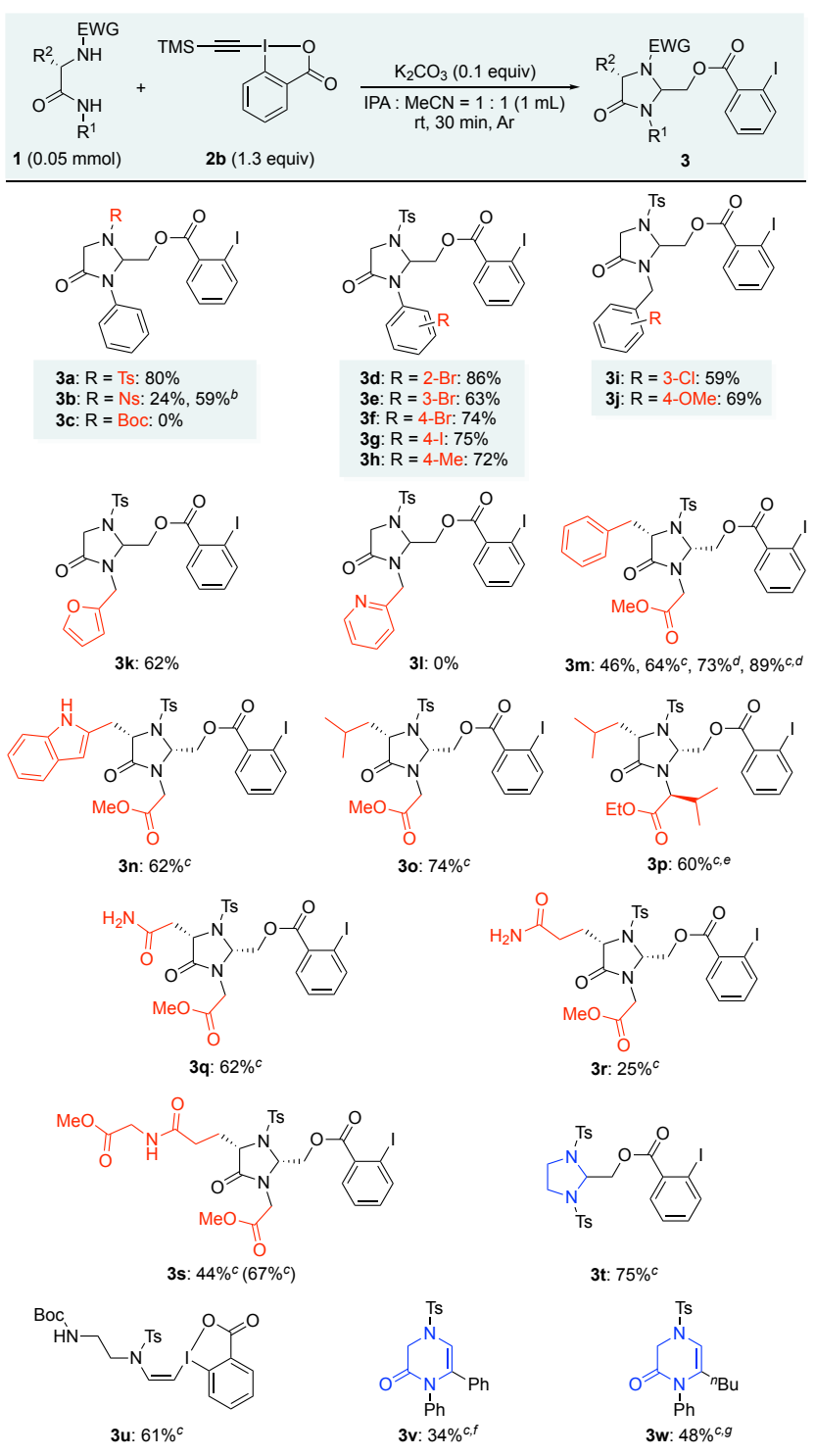

${ }^{a}$ Reaction conditions: $\mathbf{1}(0.05 \mathrm{mmol}), \mathbf{2 b}(0.065 \mathrm{mmol}), \mathrm{K}_{2} \mathrm{CO}_{3}$ (0.005 mmol), 1-propanol $(0.5 \mathrm{~mL})$, acetonitrile $(0.5 \mathrm{~mL})$, room temperature, $30 \mathrm{~min}$, argon. Isolated yields. Numbers in parentheses are ${ }^{1} \mathrm{H}$ NMR yields using 1,1,2,2-tetrachloroethane as an internal standard. ${ }^{b}$ Reaction time is $24 \mathrm{~h} .{ }^{c} \mathrm{~K}_{2} \mathrm{CO}_{3}(0.05 \mathrm{mmol})$. ${ }^{d} \mathbf{2 a}$ was used instead of $\mathbf{2 b} .{ }^{e} \mathbf{1 p}(11 \%)$ was recovered. ${ }^{f} \mathrm{Ph}-\mathrm{EBX}(\mathbf{2 c})$ was used instead of $\mathbf{2 b} .{ }^{g n} \mathrm{Bu}-\mathrm{EBX}$ (2d) was used instead of $\mathbf{2} \mathbf{b}$.

benziodoxolone, and intramolecular cyclization gave the 4imidazolidinone. Interestingly, phenyl- and butyl-substituted EBX (2c, 2d) gave no 4-imidazolidines, and 3,4-dihydropyrazin-2-one ${ }^{23}$ was obtained by regioselectively, albeit in moderate yield, probably via an ynamide intermediate $(\mathbf{3 v}, \mathbf{3 w}){ }^{16 \mathrm{a}}$ These results indicated that the use of an unsubstituted EBX reagent is essential for the successful synthesis of 4-imidazolidinones in this system. Note that these 4-imidazolidinones are all novel compounds.

It is noteworthy that glycine-derived 4-imidazolidinone 3a was further derivatized (Scheme 3). Alcohol 4 was obtained by solvolysis in the presence of $\mathrm{K}_{2} \mathrm{CO}_{3}$ in $\mathrm{MeOH} .{ }^{24}$ Furthermore, the Sonogashira reaction was conducted at the 2-iodobenzoic

Scheme 3. Derivatization of 4-imidazolidinone

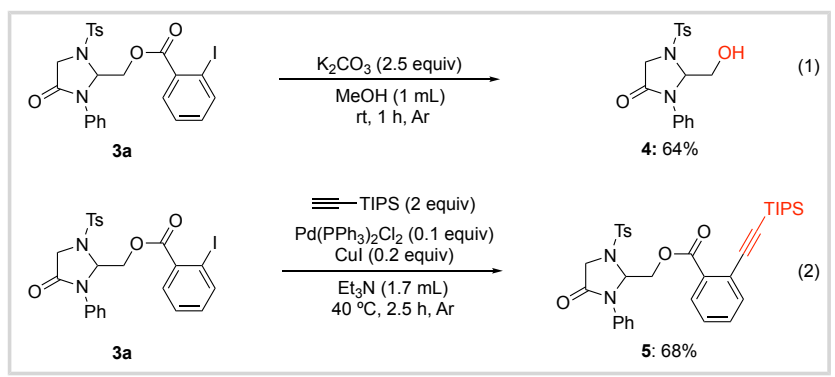

Scheme 4. Control experiment 


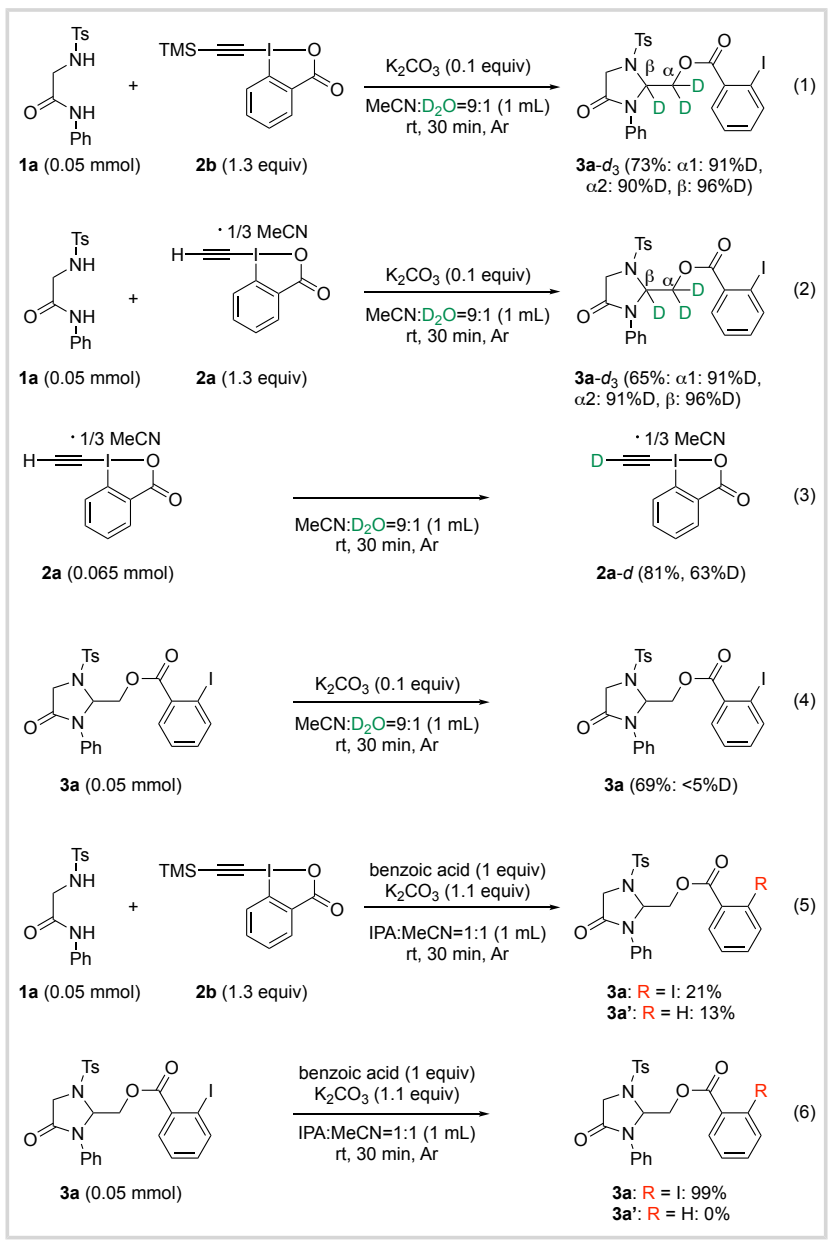

acid moiety to give alkyne $\mathbf{5}^{20 \mathrm{~b}}$

Several experiments were conducted with 1a to elucidate the reaction mechanism. Upon reaction using $\mathrm{MeCN} / \mathrm{D}_{2} \mathrm{O}=9: 1$ as a solvent, $\mathbf{2 b}$ afforded 4 -imidazolidinone $\left(\mathbf{3 a}-d_{3}\right)$ in good yield with high deuterium incorporation (equation 1 , Scheme 4). Note that deuterium was not incorporated at the $\alpha$-position of the carbonyl group of the amide (equation 1, Scheme 4). Furthermore, 2a gave a similar result (equation 2, Scheme 4). Interestingly, 2a gave $\mathbf{2 a}-d$ in $81 \%$ yield with $63 \%$ deuterium incorporation, even in the absence of a base, due to the highly electron-withdrawing nature of the $\lambda^{3}$-iodanyl group (equation 3 , Scheme 4). Conversely, when 3a was used as the substrate under the same conditions, no deuterium was incorporated (equation 4, Scheme 4). These results indicated that $\mathbf{2 b}$ converts to EBX upon deprotection of the TMS group via the relatively

Scheme 5. Plausible reaction mechanism

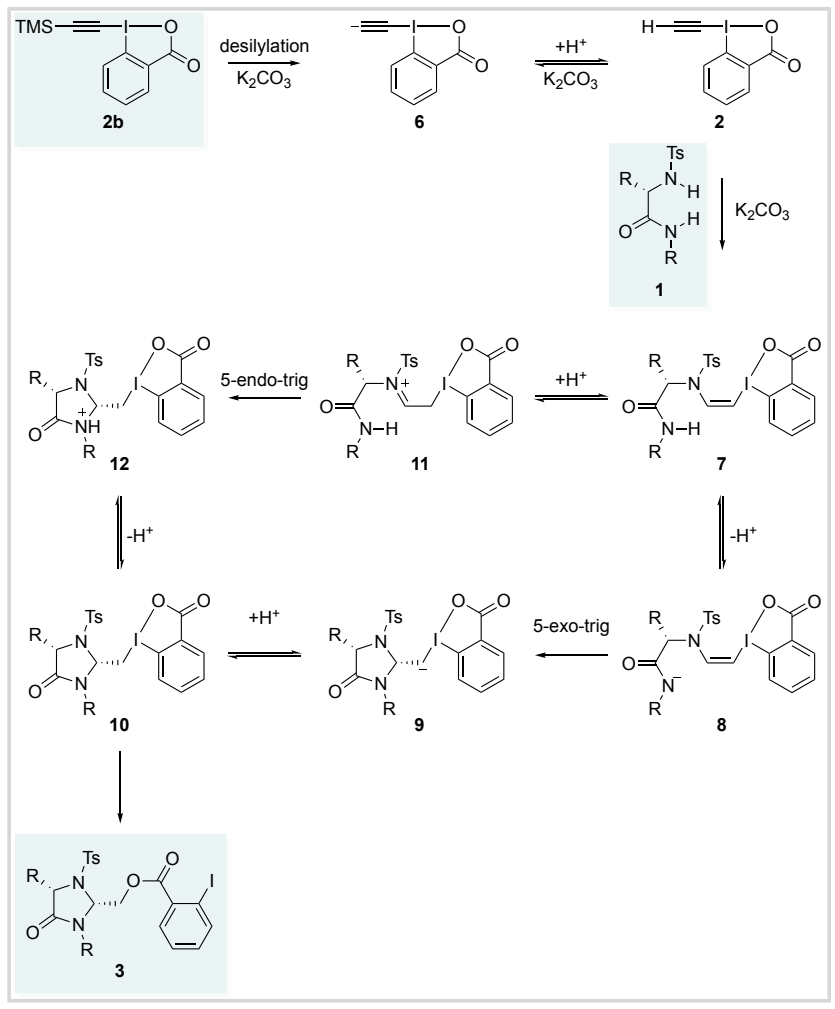

stable acetylide anion of EBX. Furthermore, the acetylenic hydrogen of 2a was rapidly abstracted in the presence of catalytic amounts of $\mathrm{K}_{2} \mathrm{CO}_{3}$ prior to the conjugate addition of $\mathbf{1 a}$ to give the trideuterated product $\mathbf{3 a}-d_{3}$. Furthermore, when we performed the reaction in the presence of benzoic acid, a mixture of the expected products 3a and 3a' was obtained (equation 5, Scheme 4). In contrast, $\mathbf{3 a}$ gave no $\mathbf{3 a}$ ' under the same conditions (equation 6, Scheme 4), indicating that intermolecular substitution of an alkyl benziodoxolone intermediate by benzoic acid in the presence of the base was a likely reaction pathway to give the 4-imidazolidinone 3a'.

Based on these results and those from previous studies, a plausible mechanism for the synthesis of 4-imidazolidinone was formulated (Scheme 5). TMS-EBX (2b) is deprotected to EBX in the presence of $\mathrm{K}_{2} \mathrm{CO}_{3}$ due to the high stability of the acetylide anion (6). The sulfonamide moiety of diamide $\mathbf{1}$ adds to the $\beta$-carbon of 2 in the presence of $\mathrm{K}_{2} \mathrm{CO}_{3}$ because of the higher acidity of the sulfonamide than that of the amide to form cis-VBX 7, probably through the coordination of the sulfonamide to the iodine center. ${ }^{18 a}$ Regrettably, we could not isolate the corresponding VBX from 1a-1t; however, the formation of $3 \mathbf{u}$ evidenced the formation of VBX as an intermediate. For the cyclized intermediate 10, 5-endo-trig and/or 5-exo-trig, cyclization is probably possible. To gain further insight into the reaction mechanism of the cyclization step, density functional theory (DFT) calculations were performed using Gaussian16, Revision C. $01 .^{25}$ The possible mechanistic routes were analyzed in terms of solvation-corrected Gibbs free energies. The results demonstrated that 5-exo-trig cyclization from $\mathbf{8}$ to $\mathbf{9}$, which is a favored mode by Baldwin's rules,${ }^{26}$ has a lower free energy barrier $\left(\Delta G^{\ddagger}=13.8 \mathrm{kcal} \mathrm{mol}^{-1}\right)$ than that of 5-endo-trig cyclization from 11 to $12\left(\Delta G^{\ddagger}=20.8 \mathrm{kcal} \mathrm{mol}^{-1}\right)$ (Figure S6). In addition, the energy difference between the deprotonation of amide 7 to anion 8 was $61.2 \mathrm{kcal} \mathrm{mol}^{-1}$ lower than the protonation of 7 to cation 11, possibly due to the highly electron deficient nature of 11. Although other cyclization mechanisms, such as via a 
betaine intermediate, cannot be excluded, the formation of betaine from 7 requires $33.3 \mathrm{kcal} \mathrm{mol}^{-1}$ (see the SI for details). These results indicate that 5-exo-trig cyclization; that is, intramolecular Michael addition, was the most likely pathway. Finally, alkyl benziodoxolone $\mathbf{1 0}$ was probably converted to $\mathbf{3}$ through rapid intermolecular substitution by 2-iodobenzoic acid, probably initially generated by the decomposition of benziodoxolones, in the presence of a base due to the hyper-leavinggroup ability of the $\lambda^{3}$-iodanyl group, as indicated by the control experiment. ${ }^{27}$ However, intramolecular reductive elimination cannot be ruled out.

In conclusion, we achieved the efficient synthesis of 4-imidazolidinones from a variety of diamides by double Michael addition, a novel reaction mode for hypervalent alkynyl iodine compounds, and a formal reductive elimination sequence using in situ-generated EBX from TMS-EBX or EBX-MeCN. The highly reactive EBX enabled chemoselective intermolecular $N$ alkenylation of the sulfonamide moiety and intramolecular cyclization of the amide moiety under mild basic conditions. The reaction diastereoselectively gave cis-2,5-disubstituted 4-imidazolidinones from amino-acid-derived diamides. Furthermore, 2-[(2-iodobenzoyloxy)methyl]-4-imidazolidinone was derivatized by solvolysis and Sonogashira coupling. DFT calculations indicated that the double Michael addition mechanism is plausible. Thus, the potential of an unsubstituted EBX reagent for the synthesis of heterocycles from complex molecules and their functionalization with mild nucleophiles was demonstrated.

\section{ASSOCIATED CONTENT}

\section{Supporting Information}

The Supporting Information is available free of charge on the ACS Publications website.

Experimental procedures and characterization data (PDF).

\section{AUTHOR INFORMATION}

\section{Corresponding Authors}

Hiroyoshi Esaki - Hyogo College of Medicine, 1-1 Mukogawacho, Nishinomiya, Hyogo 663-8501, Japan; orcid.org/0000-00019507-5205; Email: esaki@hyo-med.ac.jp

Norihiro Tada - Gifu Pharmaceutical University, 1-25-4 Daigakunishi, Gifu 501-1196, Japan; orcid.org/ 0000-0003-2871-5406; Email: ntada@gifu-pu.ac.jp Akichika Itoh - Gifu Pharmaceutical University, 1-25-4 Daigakunishi, Gifu 501-1196, Japan; orcid.org/ 0000-0002-0072-2981; Email: itoha@gifu-pu.ac.jp

\section{Authors}

Ayaka Shimizu - Gifu Pharmaceutical University, 1-25-4 Daigaku-nishi, Gifu 501-1196, Japan

Atsushi Shibata - Gifu Pharmaceutical University, 1-25-4

Daigaku-nishi, Gifu 501-1196, Japan

Kazuaki Fukushima - Hyogo College of Medicine, 1-1 Mukogawa-cho, Nishinomiya, Hyogo 663-8501, Japan; orcid.org/0000-0003-3756-5876

\section{Notes}

The authors declare no competing financial interest.

\section{ACKNOWLEDGMENT}

This work was supported by JSPS KAKENHI Grant Number 19K06977 and Grants for Research from SIS (The Society of Iodine Science). The authors would like to thank Enago (www.enago.jp) for the English language review. We are deeply grateful to DAICEL Corporation for their kind assistance with the chiral HPLC separation.

\section{REFERENCES}

(1) For a recent example, see: Li, C.; Chen, P.; Li, H.; Mahmud, T.; Wu, D.; Xu, J.; Lan, W. Potential Antidiabetic Fumiquinazoline Alkaloids from the Marine-Derived Fungus Scedosporium apiospermum F41-1. J. Nat. Prod. 2020, 83, 1082-1091.

(2) For a recent example, see: Aboul-Enein, M. N.; El-Azzouny, A. A. S.; Saleh, O. A.; Amin, K. M.; Maklad, Y. A.; Hassan, R. M. Synthesis and Anticonvulsant Activity of Substituted-1,3-diazaspiro[4.5]decan-4-ones. Arch. Pharm. 2015, 348, 575-588.

(3) For selected examples, see: (a) Adebomi, V.; Cohen, R. D.; Wills, R.; Chavers, H. A. H.; Martin, G. E.; Raj, M. CyClick Chemistry for the Synthesis of Cyclic Peptides. Angew. Chem., Int. Ed. 2019, 58, 19073-19080. (b) Rinnová, M.; Nefzi, A.; Houghten, R. A. Opioid Activity of 4-Imidazolidinone Positional Analogues of Leu-Enkephalin. Bioorg. Med. Chem. Lett. 2002, 12, 3175-3178. (c) Heck, A. J. R.; Bonnici, P. J.; Breukink, E.; Morris, D.; Wills, M. Modification and Inhibition of Vancomycin Group Antibiotics by Formaldehyde and Acetaldehyde. Chem. Eur. J. 2001, 7, 910-916.

(4) For a review, see: Lerais, G.; MacMillan, D. W. C. Modern Strategies in Organic Catalysis: The Advent and Development of Iminium Activation. Aldrichimica Acta 2006, 39, 79-87.

(5) For a recent example, see: Harmand, L.; Drabina, P.; Pejchal, V.; Husáková, L.; Sedlák, M. Recyclable catalyst for the asymmetric Henry reaction based on functionalized imidazolidine-4-one-copper(II) complexes supported by a polystyrene copolymer. Tetrahedron Lett. 2015, 56, 6240-6243.

(6) For a recent example, see: Abas, H.; Mas-Roselló, J.; Amer, M. M.; Durand, D. J.; Groleau, R. R.; Fey, N.; Clayden, J. Asymmetric and Geometry-Selective $\alpha$-Alkenylation of $\alpha$-Amino Acids. Angew. Chem., Int. Ed. 2019, 58, 2418-2422.

(7) For a review, see: Blackmore, T. R.; Thompson, P. E. Imidazolidin-4-ones: Their Syntheses and Applications. Heterocycles 2011, 83, 1953-1975.

(8) For selected examples, see: (a) Zhou, Z.; Chen, S.; Qin, J.; Nie, X.; Zheng, X.; Harms, K.; Riedel,R.; Houk, K. N.; Meggers, E. Catalytic Enantioselective Intramolecular $\mathrm{C}\left(\mathrm{sp}^{3}\right)-\mathrm{H}$ Amination of 2-Azidoacetamides Angew. Chem., Int. Ed. 2019, 58, 1088-1093. (b) Kanyiva, K. S.; Tane, M.; Shibata, T. Iodine-Catalyzed Synthesis of Chiral 4Imidazolidinones Using $\alpha$-Amino Acid Derivatives via Dehydrogenative $\mathrm{N}-\mathrm{H} / \mathrm{C}\left(\mathrm{sp}^{3}\right)-\mathrm{H}$ Coupling. J. Org. Chem. 2019, 84, 12773-12783.

(9) For selected examples, see: (a) Trindade, A. F.; Bode, J. W. Irreversible Conjugation of Aldehydes in Water To Form Stable 1,2,4Oxadiazinan-5-ones. Org. Lett. 2016, 18, 4210-4213. (b) Satz, A. L.; Cai, J.; Chen, Y.; Goodnow, R.; Gruber, F.; Kowalczyk, A.; Petersen, A.; Naderi-Oboodi, G.; Orzechowski, L.; Strebel, Q. DNA Compatible Multistep Synthesis and Applications to DNA Encoded Libraries. Bioconjugate Chem. 2015, 26, 1623-1632.

(10) For reviews on hypervalent iodine compounds, see: (a) The Chemistry of Hypervalent Halogen Compounds; Olofsson, B.; Marek, I.; Rappoport, Z. Eds.; Patai's Chemistry of Functional Groups; John Wiley \& Sons, Ltd., 2018. (b) Yoshimura, A.; Zhdankin, V. V. Advances in Synthetic Applications of Hypervalent Iodine Compounds. Chem. Rev. 2016, 116, 3328-3435.

(11) For a review on alkynyl hypervalent iodine compounds, see: Brand, J. P.; Waser, J. Electrophilic Alkynylation: The Dark Side of Acetylene Chemistry. Chem. Soc. Rev. 2012, 41, 4165-4179.

(12) For high electron-withdrawing nature, see: Mironova, A. A.; Maletina, I. I.; Iksanova, S. V.; Orda, V. V.; Yagupol'skii, L. M. Electronic Nature of Substituents Containing Polyvalent Iodine. Zh. Org. Khim. 1989, 25, 306-311.

(13) For hyper-leaving-group character, see: Okuyama, T.; Takino, T.; Sueda, T.; Ochiai, M. Solvolysis of Cyclohexenyliodonium Salt, a New Precursor for the Vinyl Cation: Remarkable Nucleofugality of the Phenyliodonio Group and Evidence for Internal Return from an Intimate Ion-Molecule Pair. J. Am. Chem. Soc. 1995, 117, 3360-3367. 
(14) For reviews on cyclic hypervalent iodine compounds, see: (a) Hari, D. P.; Caramenti, P.; Waser, J. Cyclic Hypervalent Iodine Reagents: Enabling Tools for Bond Disconnection via Reactivity Umpolung. Acc. Chem. Res. 2018, 51, 3212-3225. (b) Hari, D. P.; Nicolai, S.; Waser, J. Patai's Chemistry of Functional Groups: Alkynylations and Vinylations; eds Marek, I.; Olofsson, B.; Rappoport. Z. John Wiley \& Sons, Ltd.: 2018. (c) Li, Y.; Hari, D. P.; Vita, M. V.; Waser, J. Cyclic Hypervalent Iodine Reagents for Atom-Transfer Reactions: Beyond Trifluoromethylation. Angew. Chem., Int. Ed. 2016, 55, 4436-4454.

(15) For selected examples with metal catalysts, see: (a) Wu, J.; Yoshikai, N. Modular Synthesis of Multisubstituted Furans through Palladium-Catalyzed Three-Component Condensation of Alkynylbenziodoxoles, Carboxylic Acids, and Imines. Angew. Chem., Int. Ed. 2015, 54, 11107-11111. (b) Lu, B.; Wu, J.; Yoshikai, N. Palladium-Catalyzed Condensation of N-Aryl Imines and Alkynylbenziodoxolones To Form Multisubstituted Furans J. Am. Chem. Soc. 2014, 136, 11598 11601.

(16) For selected examples without metal catalysts, see: (a) Aubineau, T.; Cossy, J. Chemoselective alkynylation of N-sulfonylamides versusamides and carbamates - Synthesis of tetrahydropyrazines. Chem. Commun. 2013, 49, 3303-3305. (b) Liu, B.; Alegre-Requena, J. V.; Paton, R. S.; Miyake, G. M. Unconventional Reactivity of Ethynylbenziodoxolone Reagents and Thiols: Scope and Mechanism. Chem. Eur. J. 2020, 26, 2386-2394. (c) Li, M.; Li, W.; Lin, C.; Wang, J.; Wen, L. One Base for Two Shots: Metal-Free Substituent-Controlled Synthesis of Two Kinds of Oxadiazine Derivatives from Alkynylbenziodoxolones and Amidoximes. J. Org. Chem. 2019, 84, 6904-6915.

(17) For a review on vinyl benziodoxolones, see: Declas, N.; Pisella, G.; Waser, J. Vinylbenziodoxol(on)es: Synthetic Methods and Applications. Helv. Chim. Acta 2020, 103, e2000191.

(18) For selected examples on vinyl benziodoxolones, see: (a) Caramenti, P.; Declas, N.; Tessier, R.; Wodrich, M. D.; Waser, J. Stereoselective synthesis of alkyl-, aryl-, vinyl- and alkynyl-substituted Zenamides and enol ethers. Chem. Sci. 2019, 10, 3223-3230. (b) Wu, J.; Deng, X.; Yoshikai, N. Stereocontrolled Synthesis of Halovinylbenziodoxoles by Hydro- and Iodochlorination of Ethynylbenziodoxoles. Chem. - Eur. J. 2019, 25, 7839-7842.

(19) (a) Shimbo, D.; Shibata, A.; Yudasaka, M.; Maruyama, T.; Tada, N.; Uno, B.; Itoh, A. Synthesis of cis- $\beta$-Amidevinyl Benziodoxolones from the Ethynyl Benziodoxolone-Chloroform Complex and Sulfonamides. Org. Lett. 2019, 21, 9769-9773. (b) Shimbo, D.; Maruyama, T.; Tada, N.; Itoh, A. N-Alkenylation of hydroxamic acid derivatives with ethynyl benziodoxolone to synthesize cis-enamides through vinyl benziodoxolones. Org. Biomol. Chem. 2021, 19, 2442-2447. (c) Takai, R.; Shimbo, D.; Tada, N.; Itoh, A. Ligand-Enabled Copper-Catalyzed NAlkynylation of Sulfonamide with Alkynyl Benziodoxolone: Synthesis of Amino Acid-Derived Ynamide. J. Org. Chem. 2021, 86, 4699-4713. (d) Ura, T.; Shimbo, D.; Yudasaka, M.; Tada, N.; Itoh, A. Synthesis of Phenol-Derived cis-Vinyl Ethers Using Ethynyl Benziodoxolone. Chem. - Asian J. 2020, 15, 4000-4004. (e) Yudasaka, M.; Shimbo, D.; Maruyama, T.; Tada, N.; Itoh, A. Synthesis, Characterization, and Reactivity of an Ethynyl Benziodoxolone (EBX)-Acetonitrile Complex. Org. Lett. 2019, 21, 1098-1102.

(20) For selected examples, see: (a) Boelke, A.; Finkbeiner, P.; Nachtsheim , B. J. Atom-economical group-transfer reactions with hypervalent iodine compounds. Beilstein J. Org. Chem. 2018, 14, 1263 1280. (b) Borrel, J.; Pisella, G.; Waser, J. Copper-Catalyzed Oxyalkynylation of $\mathrm{C}-\mathrm{S}$ Bonds in Thiiranes and Thiethanes with Hypervalent Iodine Reagents. Org. Lett. 2020, 22, 422-427.

(21) For a review, see: Guo, H.; Fan, Y. C.; Sun, Z.; Wu, Y.; Kwon, O. Phosphine Organocatalysis. Chem. Rev. 2018, 118, 10049-10293.

(22) For a review, see: Minehan, T. G. Tandem Bond-Forming Reactions of 1-Alkynyl Ethers. Acc. Chem. Res. 2016, 49, 1168-1181.

(23) For a recent example, sees: Icelo-Ávila, E.; Amador-Sánchez, Y. A.; Polindara-García, L. A.; Miranda, L. D. Synthesis of 6-methyl3,4-dihydropyrazinones using an Ugi 4-CR/allenamide cycloisomerization protocol. Org. Biomol. Chem. 2017, 15, 360-372.

(24) Egami, H.; Kawamura, S.; Miyazaki, A.; Sodeoka, M. Trifluoromethylation Reactions for the Synthesis of $\beta$-Trifluoromethylamines. Angew. Chem., Int. Ed. 2013, 52, 7841-7844.

(25) Frisch M. J. et al., Gaussian16; Gaussian, Inc.: Wallingford, CT, 2016 (see SI for full list of authors).

(26) For Baldwin's rule, see: Baldwin, J. E. Rules for ring closure. J. Chem. Soc., Chem. Commun. 1976, 734-736.

(27) For some recent examples, see: (a) Muñiz, K.; Barreiro, L.; Romero, R. M.; Martínez, C. Catalytic Asymmetric Diamination of Styrenes J. Am. Chem. Soc. 2017, 139, 4354-4357. (b) Bosnidou, A. E.; Muñiz, K. Intermolecular Radical C $\left(\mathrm{sp}^{3}\right)-\mathrm{H}$ Amination under Iodine Catalysis, Angew. Chem., Int. Ed. 2019, 58, 7485-7489. (c) Deng, G.; Luo, J. Silver(I)-catalyzed reaction of terminal alkynes with (diacetoxyiodo) benzene: a convenient, efficient and clean preparation of $\alpha$ acetoxy ketones. Tetrahedron 2013, 69, 5937-5944. 